\title{
Characterization and Calibration of a Viscoelastic Simplified Potential Energy Clock Model for Inorganic Glasses
}

\author{
Robert S. Chambers, ${ }^{1}$ Rajan Tandon ${ }^{2}$ and Mark E. Stavig ${ }^{3}$ \\ ${ }^{1}$ Engineering Sciences Center \\ Sandia National Laboratories, Albuquerque, NM 87185-0346 \\ E-mail: rschamb@sandia.gov \\ ${ }^{2}$ Analytical Technologies Department \\ Sandia National Laboratories, Albuquerque, NM 87185-0958 \\ E-mail: rtandon@sandia.gov \\ ${ }^{3}$ Materials Science and Engineering Center \\ Sandia National Laboratories, Albuquerque, NM 87185-0958 \\ E-mail:mestavi@sandia.gov
}

\begin{abstract}
$\underline{\text { Abstract }}$
To analyze the stresses and strains generated during the solidification of glassforming materials, stress and volume relaxation must be predicted accurately. Although the modeling attributes required to depict physical aging in organic glassy thermosets strongly resemble the structural relaxation in inorganic glasses, the historical modeling approaches have been distinctly different. To determine whether a common constitutive framework can be applied to both classes of materials, the nonlinear viscoelastic simplified potential energy clock (SPEC) model, developed originally for glassy thermosets, was calibrated for the Schott 8061 inorganic glass and used to analyze a number of tests. A practical methodology for material characterization and model calibration is discussed, and the structural relaxation mechanism is interpreted in the context of SPEC model constitutive equations. SPEC predictions compared to inorganic glass data collected from thermal strain measurements and creep tests demonstrate the ability to achieve engineering accuracy and make the SPEC model feasible for engineering applications involving a much broader class of glassy materials.
\end{abstract}

Address correspondence to Robert S. Chambers, Sandia National Laboratories, P. O. Box 5800, Albuquerque, NM 87185-0346, USA.

E-mail: rschamb@sandia.gov Phone (505) 844-0771

Keywords: glasses; viscoelasticity; relaxation; stresses; modeling; strains 


\section{Introduction}

The extensive use of glass in optics, flat panel displays and glass-to-metal seals requires a knowledgeable way to manage the dimensional changes and tensile residual stresses induced by thermal processing. Thermal gradients, compaction and mismatches in thermal strains generated during cooling through the glass transition can produce unacceptable optical distortions or fractures. Although engineering analyses can provide useful information for designing robust manufacturing processes, these predictions must come from physically based material models that have been accurately calibrated and validated for the specific glasses of interest.

There are two important relaxation mechanisms that contribute to the solidification behavior in a cooling glass. The first arises from the intrinsic viscoelasticity creating a fading memory of past deformation history that eventually locks stresses in place as the viscosity increases. The second is associated with an on-going structural rearrangement of atoms as the material compacts towards the meta-stable equilibrium state of the supercooled liquid. Accurate predictions of the temporal and spatial inhomogeneities in stresses and deformations generated under general thermal processing environments must account for both mechanisms. This has been discussed by Narayanaswamy [1] in considering an analysis of the tempering process. The instant freezing approach (elastic model), stress relaxation only (viscoelastic model) and combined stress and volume relaxation (structural model) were compared, and the importance of capturing both stress and volume relaxation was demonstrated clearly.

These relaxation phenomena are not unique to inorganic glasses. Organic glassy thermosets physically age in a similar manner exhibiting both stress and volume relaxation over time. Furthermore, the basic modeling attributes required to analyze the stresses and strains generated in a polymer transitioning from the rubbery state to the glassy state are common to those needed to analyze the solidification of an inorganic glass. From a modeling perspective, it would be quite attractive to be able to implement a single constitutive framework in a finite element analysis code to predict the behavior of both material families, requiring only different sets of input parameters.

In the present work, the nonlinear viscoelastic simplified potential energy clock (SPEC) model [2] developed and validated for stress analyses of glassy thermosets is applied to an inorganic glass. In Section 2, the SPEC constitutive equations are summarized and compared to the structural relaxation model developed by Narayanaswamy [3]. Key features of the formalisms governing relaxation rates and thermal strains are discussed including a description of the competing mechanisms for predicting structural relaxation. Section 3 presents a coupled experimental and modeling approach for calibrating the SPEC constitutive equations and demonstrates its applicability to an inorganic sealing glass. The characterization data and model fitting results are presented along with comparisons between SPEC predictions and validation 
data collected from creep and volume relaxation tests conducted under complex temperature histories. The experiments, data and model predictions are discussed in Section 4 and conclusions are drawn in Section 5.

\section{Theory and Calculation}

\subsection{Linear viscoelasticity with changing time scales}

Typically, stress relaxation is defined by a stress relaxation modulus computed from the time-varying stress that results following the imposition of a step strain at a fixed temperature, Tref. In the case of simple shear, the shear stress relaxation modulus, $\mathrm{G}$, is written as

$$
G\left(t, T_{r e f}\right)=\left.\frac{\sigma_{x y}(t)}{\Delta \gamma}\right|_{\text {Tref }}
$$

where $\sigma_{x y}$ is the shear stress and $\Delta \gamma$ is the step change in shear strain. For a linear viscoelastic material, the Boltzmann superposition principle can be used to compute the contributions to the total stress from successive, multiple step strains applied at times $t_{1}$, $\mathrm{t}_{2}$, and so on. In the limit, this leads to the classic hereditary stress integral:

$$
\begin{aligned}
\sigma_{x y}(t) & =H\left(t-t_{1}\right) G\left(t-t_{1}\right) \Delta \gamma_{1}+H\left(t-t_{2}\right) G\left(t-t_{2}\right) \Delta \gamma_{2}+\ldots \\
& \rightarrow \int_{0}^{t} d s G(t-s) \frac{d \gamma}{d s} \text { in the limit }
\end{aligned}
$$

where $\mathrm{H}$ is the Heaviside step function.

Although Equation (2) suggests a way to compute the isothermal stresses in a viscoelastic material, it does not address the response of materials subjected to changing temperatures. That insight was provided by the time-temperature equivalence hypothesis of Leadermann [4]. It was observed that for many materials the "shape" of the relaxation function does not change with temperature. Instead, the function merely shifts along the logarithmic time scale as illustrated in Figure 1 where higher temperatures produce faster relaxations. This observation provided a convenient way to predict the stress relaxation modulus at different temperatures by using a new "material time", $t^{*}$ (denoted by the asterisk superscript). The new material time is computed from the real time using a socalled shift function, $\phi(T)$, or its inverse the shift factor, $a(T)=1 / \phi(T)$. For constant temperatures, $\mathrm{t}^{*}=\phi(\mathrm{T}) \cdot \mathrm{t}=\mathrm{t} / \mathrm{a}(\mathrm{T})$. Using the material time and the stress relaxation 
modulus from a known reference temperature, Tref, it is then possible to compute the relaxation function at any other temperature:

$$
G(t, T)=G\left(t^{*}, T_{r e f}\right)
$$

In the general case where temperature is changing with time, the material time must be computed by integration:

$$
t^{*}=\int_{0}^{t} d s \phi(T(s))=\int_{0}^{t} \frac{d s}{a(T(s))}
$$

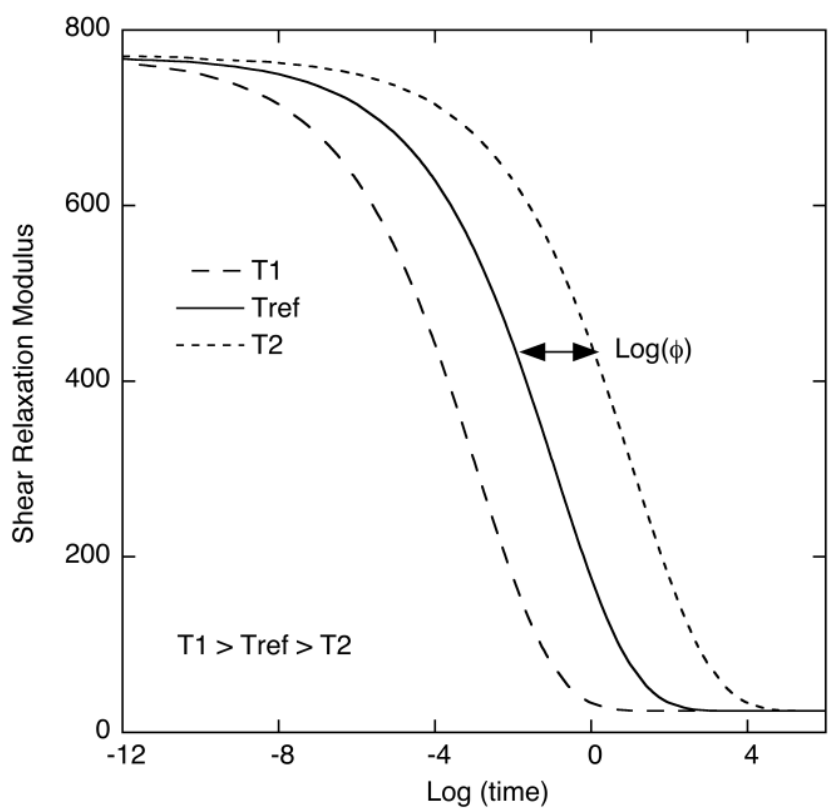

Figure 1 Plots of shear relaxation moduli at different temperatures illustrating the uniform shape of the relaxation function and the shifting of curves on the log time axis as the temperature is changed

It is also important to note that if the time-temperature equivalence hypothesis is valid, then the overall shape of the stress relaxation function can be constructed by collecting the individual portions of the relaxation curves measured during isothermal tests at different temperatures and shifting them along the log time axis relative to a selected reference temperature curve. This process defines the shape of the underlying relaxation "master curve" at Tref as well as the temperature dependence of the shift function/factor. Specific details are provided in Section 3.2. Schwarzl and Staverman [5] used the term "thermorheological simple" to describe materials that behave in this way. The validity of the hypothesis for a given material is readily determined by how well the shifting procedure works. 
In the classical development of the linear viscoelastic response of isotropic materials subjected to changing temperatures, the time-temperature equivalence hypothesis of Leaderman [4] is used to construct master curves governing the bulk (volumetric) and shear (distortional) relaxation behavior. The use of these master relaxation curves, along with a material clock defining the logarithmic time-temperature shifts, provides a natural means to capture the effect of temperature history in modulating the rates of stress relaxation. Morland and Lee [6] used this formalism to investigate the viscoelastic response of thermorheologically simple materials under nonhomogeneous temperature fields. The linear viscoelastic constitutive equations for infinitesimal deformations in an isotropic, thermorheologically simple material have the tensorial form:

$$
\begin{aligned}
& \underline{\underline{\sigma}}(t)=\left[\left\{K_{g}-K_{e q}\right\} \int_{0}^{t} d s f_{1}\left(t^{*}-s^{*}\right)\left\{\frac{d I_{1}}{d s}(s)-3 \frac{d \varepsilon_{t h}}{d s}(s)\right\}\right] \underline{\underline{I}} \\
& +2\left\{G_{g}-G_{e q}\right\} \int_{0}^{t} d s f_{2}\left(t^{*}-s^{*}\right) \frac{d \underline{\underline{\varepsilon}}}{d s}(s)+\left[K_{e q}\left\{I_{1}(t)-3 \varepsilon_{t h}(t)\right\}\right] \underline{\underline{I}} \\
& +2 G_{e q} \underline{\underline{\varepsilon}}_{d e v}(t)
\end{aligned}
$$

where $\underline{\underline{\sigma}}$ is the stress tensor, $\underline{\underline{\varepsilon}}$ is the strain tensor, $\mathrm{f}_{1}$ and $\mathrm{f}_{2}$ are normalized relaxation functions varying from 1 to 0 as time increases, $\mathrm{I}_{1}$ is the trace of the strain tensor, $\underline{\underline{I}}$ is the identity tensor, $\varepsilon_{t h}$ is the linear thermal strain, $\underline{\varepsilon}_{d e v}$ is the deviatoric strain tensor, $\mathrm{G}$ is the shear modulus and $\mathrm{K}$ is the bulk modulus. By convention, the subscripts " $\mathrm{g}$ " and "eq" on the moduli denote the instantaneous glassy and long time equilibrium values, respectively. The first bracketed term in Equation (5) describes the pressure contribution to the decaying stress coming from mechanically induced volumetric strains where the thermal strain from temperature is subtracted from the total volume strain, $\mathrm{I}_{1}$. The second integral includes the distortional relaxation arising from shear. The remaining elastic terms define the equilibrium pressure and shear stress state, i.e., the stresses when the material is fully relaxed and the integrals have vanished. When applied to the solidification of a viscous fluid (e.g., inorganic glass), the equilibrium shear modulus is set to zero. The material clock typically is specified through the use of a shift function, $\varphi$, which defines the material time, $\mathrm{t}^{*}$, governing the relaxation rates in the master curves:

$$
t^{*}-s^{*}=\int_{s}^{t} d w \varphi(w)
$$


To predict the thermal strain changes resulting from an arbitrary temperature history, Narayanaswamy [3] proposed a model of structural relaxation based on Tool's concept of a fictive temperature [7]. Using the fictive temperature, $T_{f}$, as a measure of the structural state of the glass, the thermal strain change was partitioned into contributions derived from the liquid and glassy states:

$$
\varepsilon_{t h}(T(t))=\varepsilon_{t h}(T(0))+\alpha_{l}\left\{T_{f}(t)-T(0)\right\}+\alpha_{g}\left\{T(t)-T_{f}(t)\right\}
$$

where $\varepsilon_{t h}$ is the thermal strain, T is temperature and $\alpha_{g}$ and $\alpha_{l}$ are the linear thermal expansion coefficients in the glass and liquid, respectively. Figure 2 contains a plot of a cooling curve defining the fictive temperature for the strain change computed in Equation (7). Here it can be seen that the fictive temperature corresponding to the thermal strain $\varepsilon_{t h}(T(t))$ lies on the projection of the glassy slope to the supercooled liquid dashed line (defined by the $\alpha_{l}$ slope).

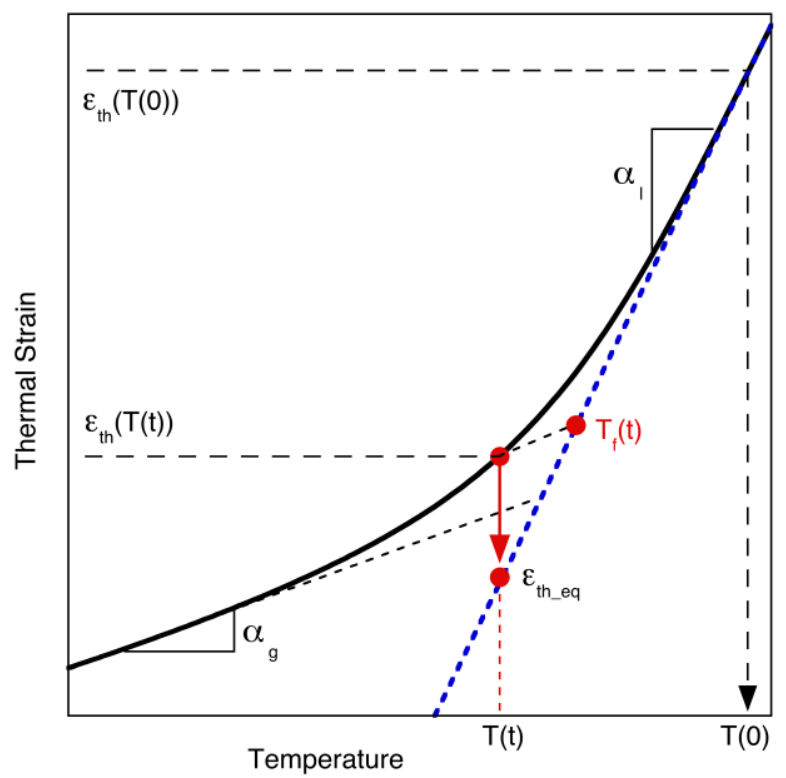

Figure 2 Plot of thermal strain change during cooling defining the fictive temperature associated with the thermal strain at temperature $\mathrm{T}(\mathrm{t})$ and illustrating the partitioning of thermal strain change into glassy and liquid portions

The governing equation for the evolution in fictive temperature is patterned after the stress relaxation formalism. In this case, the structural state of the glass is defined by a hereditary integral of the temperature history incorporating a structural relaxation modulus, $\mathrm{M}$, that varies from 1 to 0 with increasing time:

$$
T_{f}(t)=T(t)-\int_{0}^{t} d s M\left(t^{*}-s^{*}\right) \frac{d T}{d s}(s)
$$


The implications of this equation can be seen by again examining Figure 2. After reaching the strain $\varepsilon_{t h}(T(t))$ by cooling at a fixed rate, if the temperature were to be held constant, the glass would structurally relax during which time the thermal strain would decrease along the vertical path shown in Figure 2 ending at a point $\varepsilon_{t h_{-} e q}$ on the supercooled liquid line. This strain change would be realized as the integral in Equation (8) decays to zero and $\mathrm{T}_{\mathrm{f}}$ approaches $\mathrm{T}$. When fully relaxed, the total strain change would be $\alpha_{l}\{T-T(0)\}$ since $\mathrm{T}_{\mathrm{f}}=\mathrm{T}$. In the Narayanaswamy formalism, the rate of relaxation in fictive temperature is controlled by the same material clock governing stress.

Recognizing the correlation between the temperature dependence of the viscosity and relaxation times, Narayanaswamy adopted a shift function for the material clock that is fit to an equation with an Arrhenius dependence on temperature and fictive temperature through a partitioning of the activation energy:

$$
\ln (\varphi(t))=\frac{x H}{R}\left\{\frac{1}{T_{r e f}}-\frac{1}{T(t)}\right\}+\frac{(1-x) H}{R}\left\{\frac{1}{T_{r e f}}-\frac{1}{T_{f}(t)}\right\}
$$

Here Tref denotes the reference temperature for the material master curves, $\mathrm{x}$ is a partitioning factor and $\mathrm{H}$ and $\mathrm{R}$ are the activation energy and universal gas constant, respectively. This constitutive formalism has been used to analyze many problems including annealing [8], tempering [9-11], dimensional stability [12] and solidification [13] of inorganic glasses.

\subsection{Simplified Potential Energy Clock (SPEC) Model}

Glassy polymers also exhibit complex nonlinear, time-dependent relaxations in stress and volume. This has led to the development of a number of constitutive equations based on the previous assumptions of thermorheological simplicity and the use of a material clock. These clock models have been driven by a variety of different mechanisms such as free volume [14-16], entropy [17], stress [18] and strain [19]. Caruthers et al. [20] introduced a rigorous potential energy clock (PEC) model based on a Frechet series expansion of the Helmholtz free energy for materials with fading memory. This formulation enabled the clock to be based on readily computable thermodynamic quantities, which proved to be quite successful in quantitatively predicting a wide range of material behavior (e.g., stress relaxation, physical aging, creep, yielding) from a single set of model parameters [21]. Unfortunately, the PEC constitutive equations are quite complex involving four relaxation spectra, single and double hereditary integrals and a variety of temperature and volume dependencies. The simplified potential energy clock 
(SPEC) model was developed to ease the experimental and computational burdens of PEC. Although SPEC is a more phenomenological model that is easier to characterize and calibrate, its performance predictions have not been compromised [2].

For infinitesimal deformations, the SPEC viscoelastic stress equations can be written as

$$
\begin{aligned}
& \underset{=}{\sigma}(t)=\left[\left\{K_{g}-K_{e q}\right\} \int_{0}^{t} d s f_{1}\left(t^{*}-s^{*}\right) \frac{d I_{1}}{d s}(s)\right] \underline{\underline{I}} \\
& -\left[\left\{K_{g} \beta_{g}-K_{e q} \beta_{e q}\right\} \int_{0}^{t} d s f_{1}\left(t^{*}-s^{*}\right) \frac{d T}{d s}(s)\right] \underline{I} \\
& +2\left\{G_{g}-G_{e q}\right\} \int_{0}^{t} d s f_{2}\left(t^{*}-s^{*}\right) \frac{d \underline{\varepsilon}}{d s}(s)+ \\
& +\left[K_{e q}\left\{I_{1}(t)-\beta_{e q}(T(t)-T(0))\right\}\right] \underline{\underline{I}}+2 G_{e q} \underset{=d e v}{\underline{\varepsilon}}(t)
\end{aligned}
$$

where $\beta$ is the volumetric thermal expansion coefficient and again the subscripts " $\mathrm{g}$ " and "eq" denote glassy and equilibrium quantities. In general, all the integral prefactors can depend on temperature, in which case a Taylor series expansion about the reference temperature state is used to define the function. For example, $\mathrm{K}_{\mathrm{g}}(\mathrm{T})=\mathrm{K}_{\mathrm{g} \_ \text {ref }}+$ $\left(\mathrm{dK}_{\mathrm{g}} / \mathrm{dT}\right)\left(\mathrm{T}-\mathrm{T}_{\text {ref }}\right)$. Although there are similarities to Equation (5) in terms of the bulk and shear contributions to stress, there is a significant difference arising from the presence of a new hereditary integral of temperature history. Its importance will be described subsequently. The SPEC material clock definition is based on a shift factor, a, that is a function of the thermal, volumetric and deviatoric deformation histories as follows:

$$
\begin{gathered}
t^{*}-s^{*}=\int_{0}^{t} \frac{d w}{a(w)} \\
\log (a(t))=-\frac{C_{1} N(t)}{\left(C_{2}+N(t)\right)}
\end{gathered}
$$




$$
\begin{aligned}
& N(t)=\left\{\left[T(t)-T_{r e f}\right]-\int_{0}^{t} d s f_{1}\left(t^{*}-s^{*}\right) \frac{d T}{d s}(s)\right\} \\
& +C_{3}\left\{I_{1}(t)-\int_{0}^{t} d s f_{1}\left(t^{*}-s^{*}\right) \frac{d I_{1}}{d s}(s)\right\} \\
& +C_{4}\left\{\int_{0}^{t} d s f_{1}\left(t^{*}-s^{*}\right) \frac{d \varepsilon}{d s}(s)\right\}:\left\{\int_{0}^{t} d s f_{1}\left(t^{*}-s^{*}\right) \frac{\left.d \underline{\varepsilon} \frac{\varepsilon v v}{d s}(s)\right\}}{d s}\right.
\end{aligned}
$$

The quantity $\mathrm{N}$ is an approximation to the potential internal energy that drives the relaxation rates through the material clock [2]. The parameters $\mathrm{C}_{1}$ through $\mathrm{C}_{4}$ are clock constants that must be fit to data. The first two parameters are related to the WilliamsLandel-Ferry (WLF) coefficients [22], and the third constant controls the pressure dependence of the glass transition temperature. The $\mathrm{C}_{4}$ coefficient multiplies a second invariant of the effective deviatoric, viscoelastic strain history. It establishes the dependence of the material clock on the mechanical deformation and is used to drive polymer "yielding". For modeling the linear viscoelasticity of inorganic glasses, $\mathrm{C}_{3}$ and $\mathrm{C}_{4}$ are set to zero. Further details describing the derivation of these equations can be found in references [20] and [2].

\subsection{SPEC Model Mechanisms Controlling Relaxation and Thermal Strains}

There are three obvious differences between the structural relaxation model and SPEC model: 1) the material clocks have different forms, 2) the SPEC model does not use a fictive temperature for the thermal strain computation and 3) SPEC separates the temperature and volume contributions to the decaying pressure by adding a new hereditary temperature integral to the definition of stress. Ultimately, the significance of these differences must be established by comparing model predictions to experimentally measured data. However, given the past success of the structural relaxation model, it is also beneficial to determine whether the SPEC formalism is capable of making predictions similar to the structural relaxation model. This requires a closer examination of the material clock and the evolution of thermal strain with temperature history.

\subsubsection{The Material Clock}

Although the shift factor in the SPEC model is a complicated function, through a judicious choice of clock constants it can be reduced to either the familiar WLF or Arrhenius form when the material is equilibrated and the integral contributions have relaxed to zero. The SPEC clock assumes an Arrhenius form when the parameters are 
chosen such that $C_{1}=H \log (e) /\left(R T_{r e f}\right)$ and $C_{2}=\left(1+C_{3} \beta_{e q}\right) T_{r e f}$. In that case, by starting from an equilibrium, strain-free state at the reference temperature, Equation (12) simplifies to

$$
\log (a(t))=-C_{1}\left\{1-\frac{T_{r e f}}{T(t)}\right\}=-\frac{H \log (e)}{R}\left\{\frac{1}{T_{r e f}}-\frac{1}{T(t)}\right\}
$$

This result is identical to what is obtained for the equilibrated Narayanaswamy shift function in Equation (9) since the fictive temperature is equal to temperature when the material is fully equilibrated.

Similarly, when $C_{2}=\hat{C}_{2}\left(1+C_{3} \beta_{e q}\right)$ and $C_{1}=\hat{C}_{1}$, where the parameters $\hat{C}_{1}$ and $\hat{C}_{2}$ are the WLF coefficients, the equilibrated SPEC clock identically reduces to the WLF form:

$$
\begin{aligned}
\log (a(t)) & =-\left\{\frac{C_{1}\left(T(t)-T_{r e f}\right)}{C_{2} /\left(1+C_{3} \beta_{e q}\right)+\left(T(t)-T_{r e f}\right)}\right\} \\
& =-\left\{\frac{\hat{C}_{1}\left(T(t)-T_{r e f}\right)}{\hat{C}_{2}+\left(T(t)-T_{r e f}\right)}\right\}
\end{aligned}
$$

The flexibility of being able to represent both forms of the material clock is an important feature for a model that is being considered to represent a broad class of glassforming materials. How well the SPEC clock performs in glass transition must be determined by comparing predictions to data.

\subsubsection{Free thermal strain}

Perhaps the most interesting comparison between the two model formalisms comes from the methodology used to predict thermal strain under a prescribed temperature history. This is best understood by considering predictions made for an initially stress and strain free, equilibrated material that is unconstrained and subjected to a uniform temperature change. Under these conditions, no stress is generated, the deviatoric strain is zero, and the normal (principal) strains are all equal. Hence, the only unknown is the magnitude of the volumetric strain, $\mathrm{I}_{1}$. The constitutive equation for the structural relaxation model, Equation (5), can be solved for $\mathrm{I}_{1}$. 


$$
\begin{aligned}
0= & {\left[\left\{K_{g}-K_{e q}\right\} \int_{0}^{t} d s f_{1}\left(t^{*}-s^{*}\right)\left\{\frac{d I_{1}}{d s}(s)-3 \frac{d \varepsilon_{t h}}{d s}(s)\right\}\right] } \\
& +\left[K_{e q}\left\{I_{1}(t)-3 \varepsilon_{t h}(t)\right\}\right]
\end{aligned}
$$

The obtained solution is

$$
I_{1}=3 \varepsilon_{t h}
$$

Computing the thermal strain from Equations (7) and (8) gives the result that

$$
I_{1}(t)=3 \alpha_{l}[T(t)-T(0)]-3\left[\alpha_{l}-\alpha_{g}\right] \int_{0}^{t} d s M\left(t^{*}-s^{*}\right) \frac{d T}{d s}(s)
$$

where the volumetric and thermal strains were initialized to zero at time zero.

By imposing the same initial and boundary conditions on the SPEC formalism, the constitutive equation for stress becomes:

$$
\begin{aligned}
& 0=K_{g} \int_{0}^{t} d s f_{1}\left(t^{*}-s^{*}\right) \frac{d\left(I_{1}-\beta_{g} T\right)}{d s}(s) \\
& -K_{e q} \int_{0}^{t} d s f_{1}\left(t^{*}-s^{*}\right) \frac{d\left(I_{1}-\beta_{e q} T\right)}{d s}(s) \\
& +\left\{K_{e q}\left[I_{1}-\beta_{e q}(T(t)-T(0))\right]\right\}
\end{aligned}
$$

As is, this equation cannot be solved easily for the volumetric strain, $\mathrm{I}_{1}$. However, for the particular case when the bulk modulus is constant, the equation simplifies to the result:

$$
I_{1}(t)=\beta_{e q}[T(t)-T(0)]-\left[\beta_{e q}-\beta_{g}\right] \int_{0}^{t} d s f_{1}\left(t^{*}-s^{*}\right) \frac{d T}{d s}(s)
$$

Aside from differences in the material clock, this equation is identical in form to the solution obtained for the structural relaxation model, Equation (18).

Not only does this suggest the possibility of being able to capture the stress and volume relaxations of both organic and inorganic glasses with the SPEC formalism, it also explains the role and need for the structural relaxation spectrum in historical glass modeling. When the SPEC bulk modulus is assumed to be constant, the relaxation spectrum prescribed in the SPEC hereditary temperature integral can be identified as nothing more than the Narayanaswamy structural relaxation function. From a 
constitutive standpoint, the bulk and temperature contributions to stress are defined most generally by separate integrals and prefactors. In the more traditional equations of linear viscoelasticity, the temperature integral is absorbed into the bulk integral by making additional assumptions about the prefactors and relaxation functions and by defining a mechanical strain through the subtraction of thermal strain from the total volumetric strain as shown in Equation (5). In the SPEC model, the two integrals are maintained separately, but the relaxation spectrum is assumed to be common to both. This makes the structural relaxation spectrum identically equal to the bulk relaxation spectrum. By not allowing for the separate hereditary temperature integral in the general form of the constitutive equation, there is an imposed need for a new response function to fill the deficiency. In the Narayanaswamy model, that is fulfilled by the structural relaxation modulus and fictive temperature in Equation (8), and the thermal strain definition in Equation (7).

In studying the dielectric loss for five organic glasses, Hecksher, Olsen, Niss and Dyre [23] found that all the glasses had an internal clock and age consistent with the Tool-Narayanaswamy formalism. They further noted that the liquids appear to have an exponential long-time relaxation for both the dielectric and structural relaxation. This has caused researchers to question whether a common material clock with exponential long-time behavior might be evident in other relaxations (e.g., volume) as well. If true, the SPEC construct would be consistent with such a finding.

It is also worth noting that many applications of the structural relaxation model have been made under the constant bulk modulus assumption [10, 13, 24-26]. Because of data limitations created by the difficulty in measuring the bulk relaxation modulus directly, analysts using the traditional forms of linear viscoelastic equations, employing only bulk and shear hereditary integrals in stress, have appealed to one of two modeling assumptions: 1) Poisson's ratio is constant, or 2) the bulk modulus is constant. A constant Poisson's ratio gives the rather unsatisfactory result that the bulk relaxation modulus decays to zero. Alternatively, a constant bulk modulus produces the need for a structural relaxation function, another state variable (i.e., fictive temperature) and a thermal strain definition to capture the volume relaxation mechanism. Although Scherer and Rekhson published findings [27] indicating that in the absence of high volumetric constraints a constant bulk modulus is a reasonable assumption, that assumption is not necessary, and it does not eliminate the need to characterize the volume relaxation. That is required for both model formalisms.

\section{Experimental and Results}

One of the biggest impediments to viscoelastic modeling is the difficulty in obtaining material data for model calibration. These data consist of the bulk and shear moduli, thermal expansion coefficients, relaxation functions and material clock 
parameters. To evaluate the SPEC model formalism, the Schott 8061 glass was selected

for characterization and model calibration. This is an alkali-barium-silicate glass

manufactured by Schott and used by the connector industry in glass-to-metal seals to provide electrical connections for hermetically sealed components. A concerted effort was made not only to obtain data for this project but also to develop a simple and practical methodology for characterizing the viscoelasticity of other materials.

\subsection{Moduli temperature dependence}

The initial glass tests were designed to define the glass transition temperature, $\mathrm{Tg}$, and the temperature dependences of the "elastic" moduli (i.e., glassy behavior). This was accomplished by measuring the in-phase and out-of-phase responses in dynamic tests imposing an oscillatory deformation at $1 \mathrm{~Hz}$ as the temperature was ramped from $-80^{\circ} \mathrm{C}$ to $600^{\circ} \mathrm{C}$ at $2^{\circ} \mathrm{C} / \mathrm{min}$. The tests were conducted on rectangular samples taken from the bulk glass and shaped to have flat, parallel faces that were nominally $55 \mathrm{~mm}$ long with a cross-section of $11 \mathrm{~mm}$ by $1.1 \mathrm{~mm}$. Experiments were conducted in two different modes of "infinitesimal" deformations to extract the linear response. The tensile moduli were measured in a 3-point bending test using the TA Q800 Dynamic Mechanical Analyzer. The shear moduli were measured under torsion in the TA ARES rheometer. Two torsion tests and three bending tests were conducted. Each bending sample was given a different thermal conditioning to determine whether residual stresses or the structural state of the glass significantly alter the measured moduli. The three conditioning states considered were: "as received", annealed and rapidly cooled. The two torsion tests were conducted on "as received" samples. The storage moduli from these tests are plotted in Figure 3, and the loss moduli are plotted in Figure 4. 


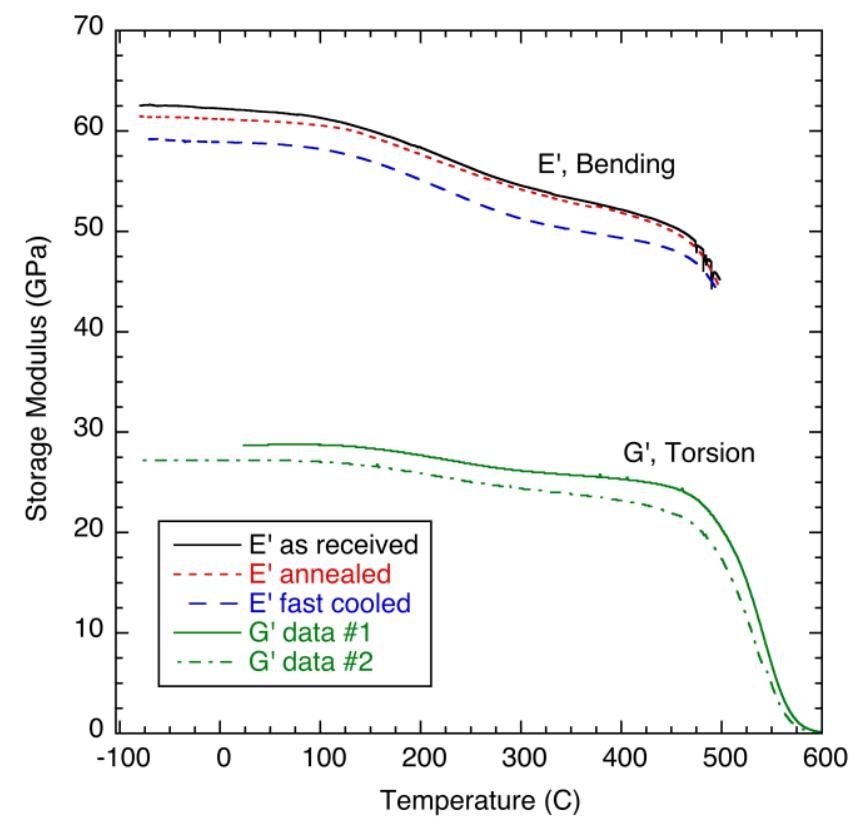

Figure 3 Temperature dependent storage moduli in S8061 glass measured in 3-point bending and torsion at $1 \mathrm{~Hz}$ ramping $2^{\circ} \mathrm{C} / \mathrm{min}$

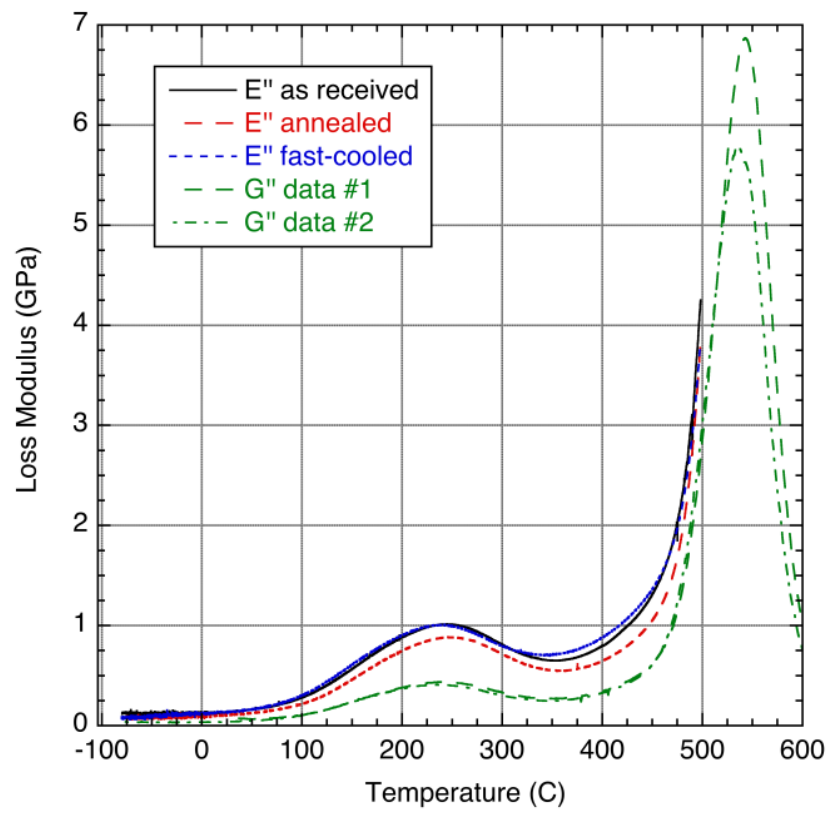

Figure 4 Temperature dependent loss moduli in S8061 glass measured in 3-point bending and torsion at $1 \mathrm{~Hz}$ ramping $2^{\circ} \mathrm{C} / \mathrm{min}$ 


\subsection{Shear master curves}

To construct the shear master curve, dynamic torsion tests were performed on similar samples under a different test protocol. Annealed samples were reheated to the starting test temperature where they were allowed to thermally equilibrate for 10 minutes. Then the shear storage and loss moduli were measured at different frequencies ranging from 0.1 to $16 \mathrm{~Hz}$. Following this, the sample temperature was changed by $10^{\circ} \mathrm{C}$, held at constant temperature for another 5 minutes and the frequency scan repeated. By continuing this process, data were collected over a temperature range from $300-600^{\circ} \mathrm{C}$. This produces a modulus vs frequency curve for each test temperature. The corresponding tan delta curves then are obtained by computing the ratio of loss modulus to storage modulus at each frequency for each temperature.

If the glass is thermorheologically simple, then it is possible to select one tan delta vs frequency curve for a chosen reference temperature, Tref, near or above the glass transition temperature, and horizontally shift all other tan delta curves in log frequency space to obtain the smooth shape of a master curve. This is the first test of the timetemperature equivalence hypothesis. The tan delta data are selected for shifting because this removes the need for any vertical shifts attributed to temperature dependence in the moduli. Once the shift factors have been defined from the tan delta curves, they can be applied to the storage and loss moduli data to construct those master curves. This is a second check on the validity of thermorheological simplicity.

To use the master curve data in a finite element analysis based on the SPEC viscoelastic constitutive equation, it is necessary to map from the frequency domain into the time domain. For numerical ease, it is advantageous to express the relaxation spectra in terms of an exponential series expansion (i.e., Prony series):

$$
G(t)=G_{g} \sum_{i=1}^{N} f_{i} \exp \left(-\frac{t}{\tau_{i}}\right)=G_{g} f(t)
$$

where the function $\mathrm{f}(\mathrm{t})$ is the normalized relaxation function that decays from 1 to 0 . When the exponential series is substituted into the shear hereditary integral of Equation (2), and the stress is computed for a sinusoidal, constant amplitude shear strain history, $\gamma_{o} \sin (\omega t)$, expressions are obtained for the storage and loss moduli in terms of the exponential series parameters and the frequency, $\omega$ :

$$
G^{\prime}(\omega)=G_{g} \sum_{i=1}^{N} f_{i} \frac{\left(\omega \tau_{i}\right)^{2}}{1+\left(\omega \tau_{i}\right)^{2}}
$$




$$
G^{\prime \prime}(\omega)=G_{g} \sum_{i=1}^{N} f_{i} \frac{\left(\omega \tau_{i}\right)}{1+\left(\omega \tau_{i}\right)^{2}}
$$

These two equations are suitable for fitting the master curves for the storage and loss moduli. Once that fit is obtained and the Prony coefficients, $\mathrm{f}_{\mathrm{i}}$ and $\tau_{i}$, are known, the relaxation modulus in the time domain is defined by Equation (21). The shifted master curve data and the corresponding Prony series fits for the storage and loss moduli are plotted in Figure 5.

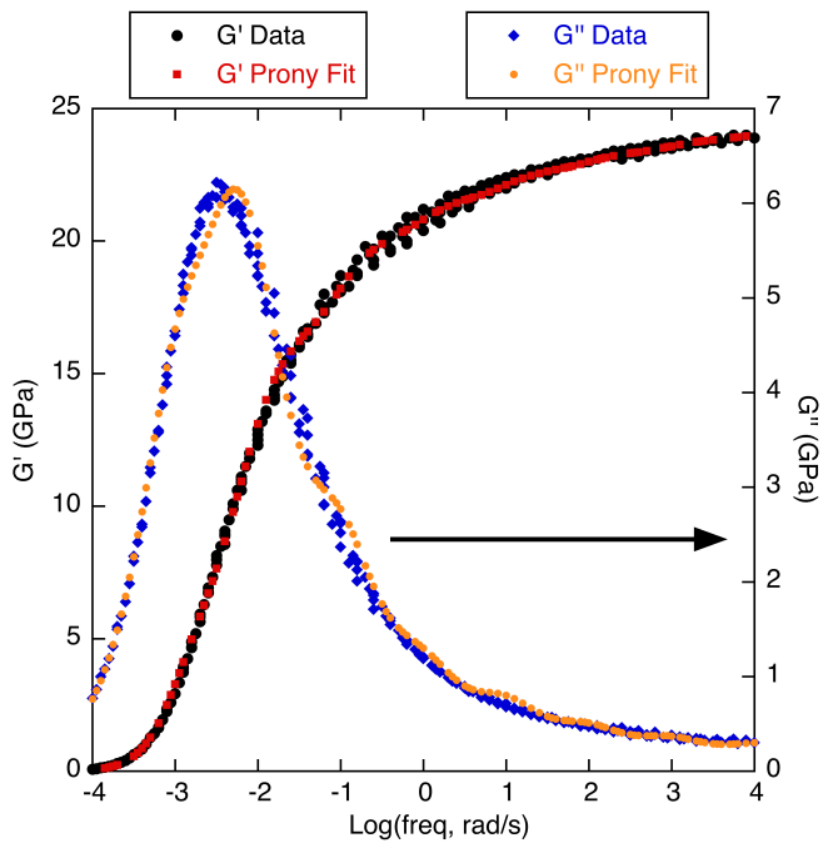

Figure 5 Shear storage and loss moduli master curve data for S8061 glass shifted to reference temperature $460^{\circ} \mathrm{C}$ and plotted with exponential series expansion fits to the master curves

The shift factors defining these master curves are plotted in Figure 6. For comparative purposes, both the WLF and Arrhenius forms of the shift factor were fit to the data. These results are provided in Equations (24) and (25), respectively. Note the temperature unit in these equations is Kelvin, but the plots are in centigrade. 


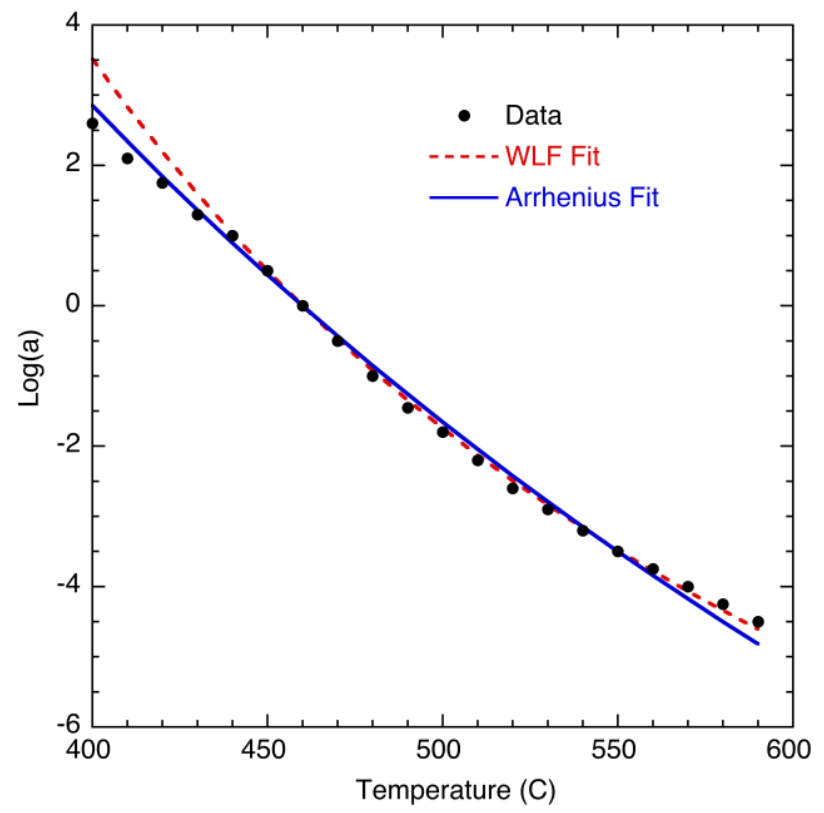

Figure $6 \log (a)$ shift factors for Tref $=460^{\circ} \mathrm{C}$ master curve construction for S8061 glass plotted with WLF and Arrhenius fits to data

$$
\begin{aligned}
& \log (a(T))=-\frac{17(T-733)}{350+(T-733)} \\
& \log (a(T))=23560\left(\frac{1}{T}-\frac{1}{733}\right)
\end{aligned}
$$

As a check on the reproducibility of these findings, another dynamic test was performed to construct a second master curve. The isothermal data were shifted and fit to the Prony series as before, and a second representation of the shear relaxation function was obtained in the time domain. In addition, a separate independent set of torque relaxation tests was performed at temperatures of $380,400,440,450$ and $460^{\circ} \mathrm{C}$. In these tests, the shear relaxation modulus was directly measured in the time domain. By shifting the temperature curves horizontally based on the $460^{\circ} \mathrm{C}$ reference temperature, a third representation of the master curve was generated. In the latter case, some small vertical shifting was required to obtain a smooth master curve. All three results are plotted together in Figure 7. 


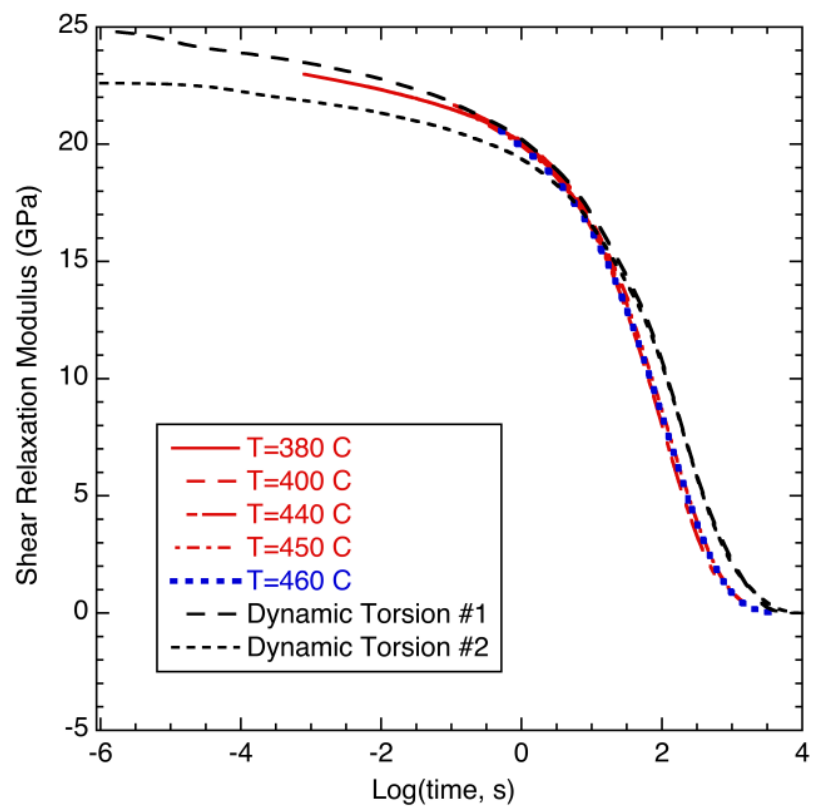

Figure 7 Shear relaxation moduli plotted at Tref $=460^{\circ} \mathrm{C}$ comparing results transformed from two oscillatory tests to shifted data measured directly in torque relaxation tests at various temperatures

\subsection{Bulk relaxation}

A direct measurement of the bulk relaxation spectrum would require imposing a step change in volume at constant temperature while measuring the decrease in pressure with time. For most laboratories, this test would be impractical given a glass transition in the $460^{\circ} \mathrm{C}$ range. Alternatively, since the time-dependent volumetric response in the SPEC equations is defined by a common relaxation spectrum, $\mathrm{f}_{1}$, employed in both the $\mathrm{I}_{1}$ and temperature hereditary integrals, thermal strain measurements provide another approach. By varying the temperature history and measuring the corresponding thermal strains, it is possible to deduce information about the bulk/volume relaxation function. This is similar to the approach taken by Rekhson and Mazurin [28] in defining the structural relaxation modulus. Since the strain measurements must be conducted within the glass transition regime on a material that is not in structural equilibrium, the SPEC constitutive equations must be used to analyze the actual test. This is handled most easily by having the SPEC model implemented in a finite element analysis code. That was the approach taken for this work using the ADAGIO Sierra software [29]. Characterization tests were conducted and analyzed, and model predictions were compared to data. Through an iterative process, the model parameters then were modified to improve predictions and converge on an acceptable set of material model properties. 
In general, five SPEC quantities must be determined to fully define the bulk/volume relaxation: both the glassy and equilibrium values of the bulk modulus and coefficients of thermal expansion as well as the bulk/thermal relaxation spectrum. The glassy and equilibrium (liquid) coefficients of thermal expansion are extracted from the thermal strain versus temperature curves measured using a dilatometer. The glassy bulk modulus can be obtained reasonably well from ultrasonic test data or computed from other elastic constants measured at room temperature. The equilibrium bulk modulus is much harder to acquire, being only attainable from the long-time tail of the relaxation spectrum. Gy proposed a way to compute the equilibrium bulk modulus from uniaxial and shear relaxation data [30]. As a starting estimate for this project, the ratio of glassy to rubbery bulk moduli was assumed to be 3.3 following the approach of Rekhson [31] based on the dilatational compliance work of Corsaro [32]. The task of determining the bulk relaxation spectrum is complicated by the need to define a function, not a scalar parameter. Although the numerical integration of the SPEC constitutive equations ultimately requires the spectrum to be represented as a Prony series, there are distinct advantages to starting with a stretched exponential where only two constants are needed to define the function:

$$
f_{1}(t)=\exp \left(-\left(\frac{t}{\tau}\right)^{\beta}\right)
$$

The relaxation time, $\tau$, positions the function on the time axis while the exponent, $\beta$, defines the width of the relaxation spectrum. By using the stretched exponential to define the functional form of the bulk relaxation modulus, the parameterization is reduced to an optimization of only two constants. After choosing initial values for $\beta$ and $\tau$, the function $\mathrm{f}_{1}$ can be fit with a Prony series (automatically done within the finite element code), and a finite element analysis of the characterization test is performed using the other estimated parameters. Based on the comparison between predictions and data, the parameters are modified, and the process is repeated. Because these are one-element problems, using the finite element software only to evaluate the constitutive equation, the turnaround is rapid and solution time is negligible.

\subsection{Thermal strain data}

To provide characterization and validation data for the structural relaxation behavior, experiments were conducted to measure the thermal strain changes generated by prescribed temperature histories. The thermal strain data were collected in a DIL 402 $\mathrm{C}$ Netzsch dilatometer using rectangular samples nominally $25 \mathrm{~mm}$ long with a $5 \mathrm{~mm}$ by $5 \mathrm{~mm}$ cross-section. The prescribed contact force was 15 centi-newtons resulting in an 
average axial stress of about $0.006 \mathrm{MPa}$. Figure 8 contains a plot of the thermal strain in an annealed sample that was heated and cooled at $0.5^{\circ} \mathrm{C} / \mathrm{min}$ between $500^{\circ} \mathrm{C}$ and room temperature multiple times. Only the first reheat and second cooling data are plotted along with the output from the SPEC model fit. Other cycles overlapped.

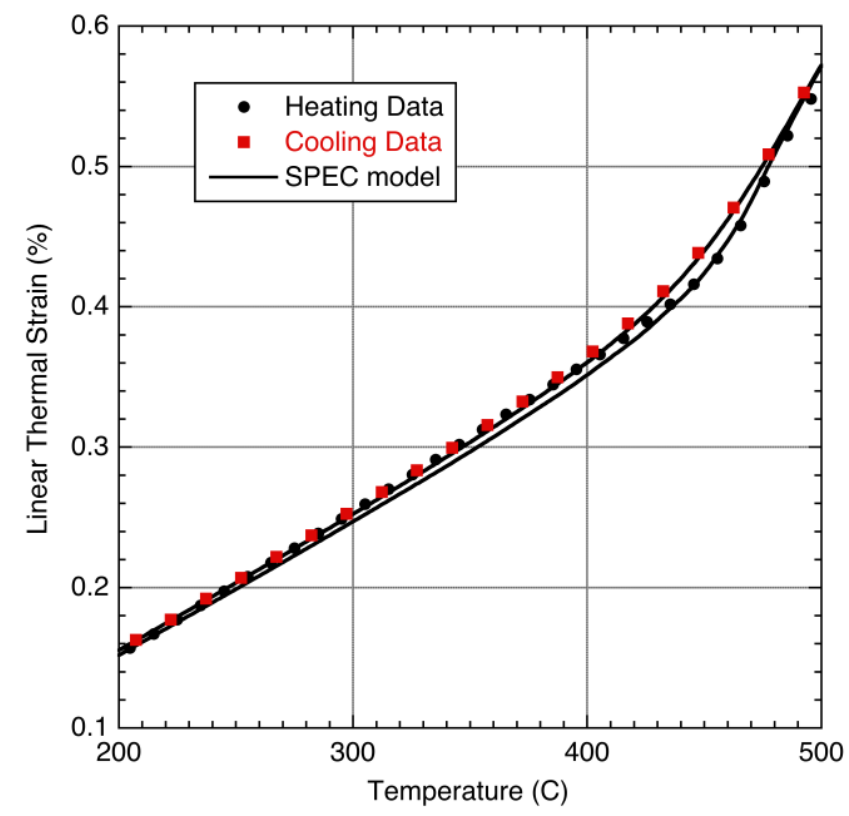

Figure 8 Plots of thermal strain data and SPEC model fit for S8061 glass annealed at $500^{\circ} \mathrm{C}$ and thermally cycled at $0.5^{\circ} \mathrm{C} / \mathrm{min}$

The second test used for calibrating the SPEC bulk/volume response prescribed a temperature history whereby the sample was annealed at $500^{\circ} \mathrm{C}$ and then rapidly cooled at $30^{\circ} \mathrm{C} / \mathrm{min}$ below glass transition and reheated at $0.5^{\circ} \mathrm{C} / \mathrm{min}$. This test was designed to probe the characteristic hysteresis generated by the additional volume relaxation that occurs during reheating at the slower rate. Test data and the model fit are compared in Figure 9. 


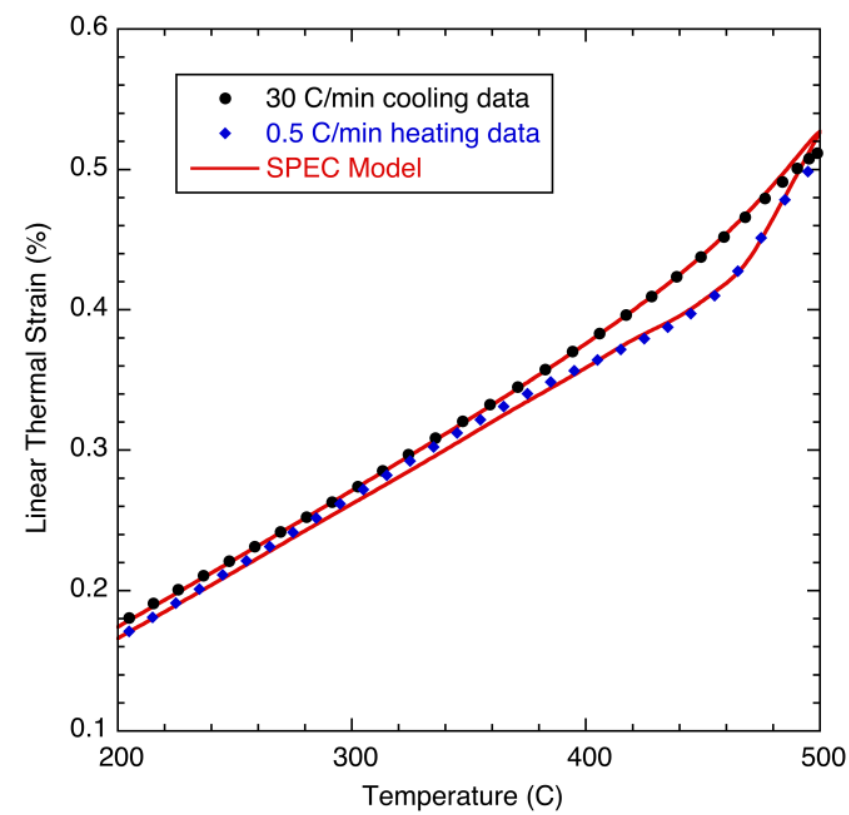

Figure 9 Plots of thermal strain data and SPEC model fit for S8061 glass annealed at $500^{\circ} \mathrm{C}$ and then rapidly cooled at $30^{\circ} \mathrm{C} / \mathrm{min}$ followed by reheating at $0.5^{\circ} \mathrm{C} / \mathrm{min}$

\subsection{SPEC model calibration}

After collecting the characterization data and calibrating the SPEC model as described in Sections 3.1-3.4, a single set of material properties was identified. The nonzero SPEC model inputs are defined in Table 1 and Table 2. Using these material definitions, additional experiments were analyzed to assess the fidelity of the SPEC predictions.

Table 1 SPEC model parameters for Schott 8061 glass at Tref $=460^{\circ} \mathrm{C}$

\begin{tabular}{|cc|}
\hline $\mathrm{K}_{\mathrm{g}}(\mathrm{GPa})$ & 33 \\
\hline $\mathrm{dK}_{\mathrm{g}} / \mathrm{dT}\left(\mathrm{MPa} /{ }^{\circ} \mathrm{C}\right)$ & -20.5 \\
\hline $\mathrm{K}_{\mathrm{eq}}(\mathrm{GPa})$ & 6 \\
\hline $\mathrm{dK}_{\mathrm{eq}} / \mathrm{dT}\left(\mathrm{MPa} /{ }^{\circ} \mathrm{C}\right)$ & -5.9 \\
\hline$\beta_{g}\left(\mathrm{ppm} /{ }^{\circ} \mathrm{C}\right)$ & 27 \\
\hline$\beta_{e q}\left(\mathrm{ppm} /{ }^{\circ} \mathrm{C}\right)$ & 90 \\
\hline $\mathrm{G}_{\mathrm{g}}(\mathrm{GPa})$ & 24.8 \\
\hline $\mathrm{dGg} / \mathrm{dT}\left(\mathrm{MPa} /{ }^{\circ} \mathrm{C}\right)$ & -7 \\
\hline $\mathrm{T}_{\mathrm{ref}}\left({ }^{\circ} \mathrm{C}\right)$ & 460 \\
\hline $\mathrm{C}_{1}$ & 17 \\
\hline
\end{tabular}




\begin{tabular}{|cc|}
\hline $\mathrm{C}_{2}$ & 350 \\
\hline $\mathrm{f}_{1}(\mathrm{t})$ & $\exp \left(-(t / 30)^{0.30}\right)$ \\
\hline
\end{tabular}

Table 2 Prony Series Coefficients for Normalized Shear Relaxation Function

\begin{tabular}{|cc|}
\hline Normalized Prony Prefactor $\mathrm{f}_{\mathrm{i}}$ & Prony Relaxation Time $\tau_{i}(\mathrm{secs})$ \\
\hline $4.24345 \mathrm{e}-3$ & $1 \mathrm{e}-8$ \\
\hline $6.63991 \mathrm{e}-3$ & $1 \mathrm{e}-7$ \\
\hline $6.55255 \mathrm{e}-3$ & $1 \mathrm{e}-6$ \\
\hline $1.41373 \mathrm{e}-2$ & $1 \mathrm{e}-5$ \\
\hline $1.40109 \mathrm{e}-2$ & $1 \mathrm{e}-4$ \\
\hline $2.01033 \mathrm{e}-2$ & $1 \mathrm{e}-3$ \\
\hline $2.66430 \mathrm{e}-2$ & $1 \mathrm{e}-2$ \\
\hline $4.31981 \mathrm{e}-2$ & $1 \mathrm{e}-1$ \\
\hline $5.98391 \mathrm{e}-2$ & $1 \mathrm{e}+0$ \\
\hline $1.46465 \mathrm{e}-1$ & $1 \mathrm{e}+1$ \\
\hline $1.62036 \mathrm{e}-1$ & $1 \mathrm{e}+2$ \\
\hline $2.57094 \mathrm{e}-1$ & $1.9953 \mathrm{e}+2$ \\
\hline $2.39037 \mathrm{e}-1$ & $1 \mathrm{e}+3$ \\
\hline
\end{tabular}

A plot of the weighted distribution in relaxation times from the normalized shear relaxation spectrum in Table 2 is provided in Figure 10. 


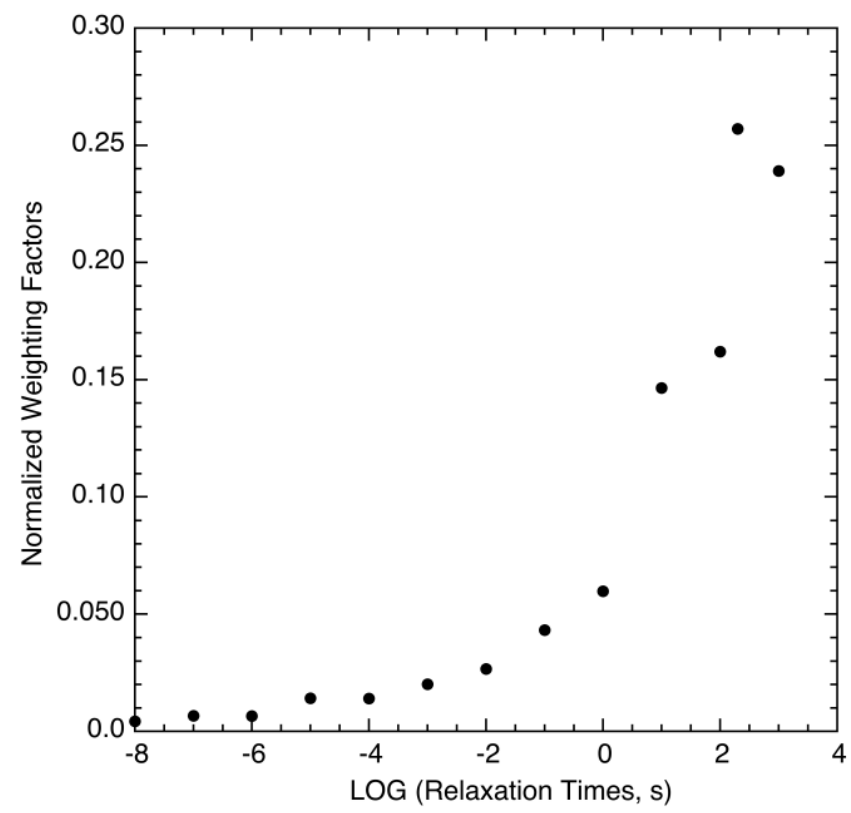

Figure 10 Plot of the normalized distribution of shear relaxation times with weighting factors fit to the shear relaxation function for the $\mathrm{S} 8061$ glass at $\mathrm{T}=460^{\circ} \mathrm{C}$

\subsection{Complex temperature histories}

Varying temperature ramps combined with temperature holds and reversals between cooling and heating pose challenges for both modeling and experiments. It is difficult for the model because the hereditary integrals and material clock must accurately account for past history. The experiments are difficult because they require adequate temperature controls along with the capability to measure small thermal strain changes during prolonged temperature holds.

Two different complex temperature histories were examined. In the first, the DIL $402 \mathrm{C}$ Netzsch dilatometer was programmed to execute the following temperature sequence: anneal at $510^{\circ} \mathrm{C}$ for 20 minutes, cool to $350^{\circ} \mathrm{C}$ at $2.5^{\circ} \mathrm{C} / \mathrm{min}$, heat to $430^{\circ} \mathrm{C}$ at $2.5^{\circ} \mathrm{C} / \mathrm{min}$, hold at $430^{\circ} \mathrm{C}$ for 3 hours, heat to $475^{\circ} \mathrm{C}$ at $6^{\circ} \mathrm{C} / \mathrm{min}$, hold at $475^{\circ} \mathrm{C}$ for 1 hour, heat to $510^{\circ} \mathrm{C}$ at $2.5^{\circ} \mathrm{C} / \mathrm{min}$, then allow to cool in the furnace to room temperature. Three such experiments were performed. A plot of the comparison between the measured and predicted strains is shown in Figure 11. 


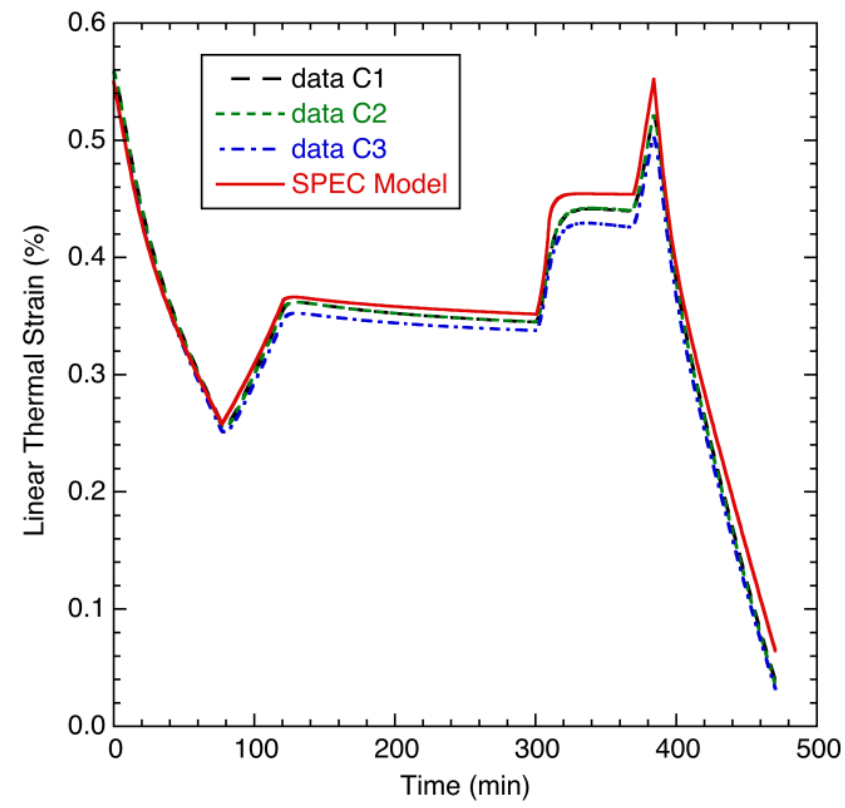

Figure 11 Comparison between measured strain and SPEC model predictions when S8061 glass was subjected to complex temperature history

A second thermal history was prescribed, consisting of a series of step temperature changes and holds to measure the volume relaxation at different temperatures. After annealing the sample at $500^{\circ} \mathrm{C}$, it was cooled to $450^{\circ} \mathrm{C}$ where the step changes were initiated. The temperature history, measured strains and SPEC model predictions are shown in Figure 12. Note the Y-axes employed in Figure 11 and Figure 12 are different reflecting differences in magnitude as well as what was taken as the zero strain state. 


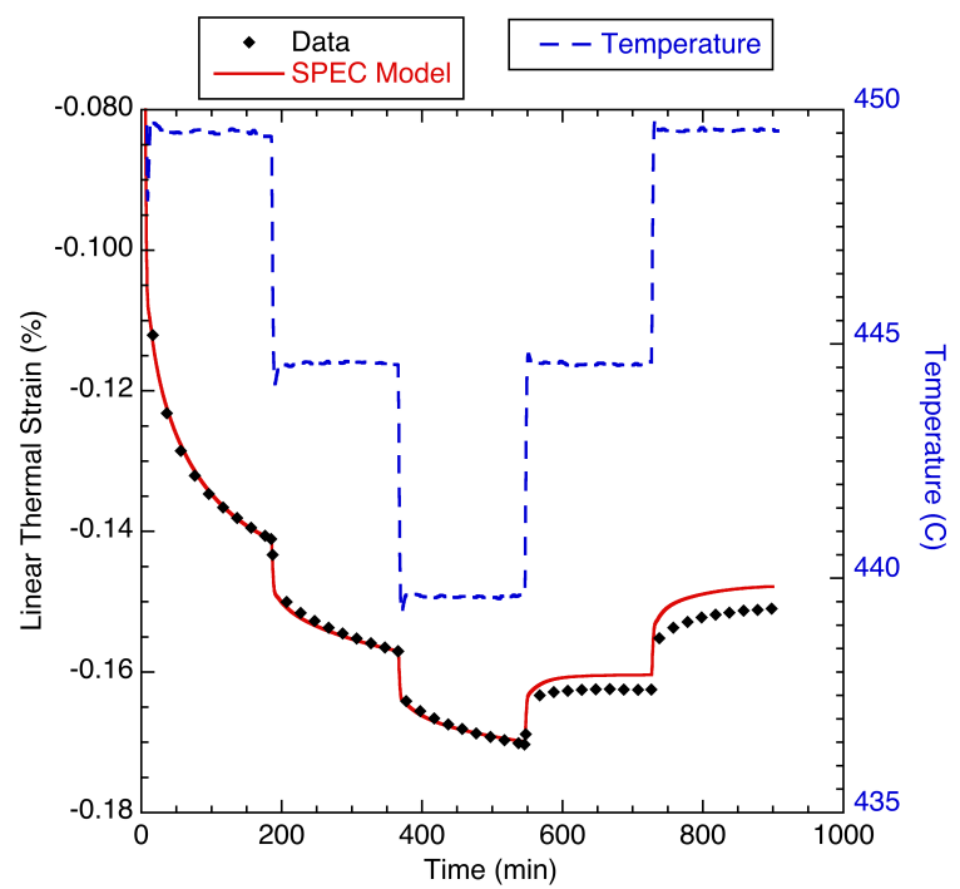

Figure 12 Comparison between measured thermal strain and SPEC model predictions for S8061 glass subjected to a series of step temperature changes and isothermal holds

\subsection{Monotonic Loading and Creep}

Two additional test types were performed to evaluate the mechanical response to loading in 3-point bending geometries. All tests were performed on the nominally sized $55 \mathrm{~mm} \times 11 \mathrm{~mm} \times 1.1 \mathrm{~mm}$ glass beams using the TA Q800 Dynamic Mechanical Analyzer. The span between supports was 50mm. In the first case, an annealed sample was reheated to $460^{\circ} \mathrm{C}$, held for 10 minutes and then loaded monotonically at $5 \mathrm{~N} / \mathrm{min}$ while measuring the center deflection. The data and SPEC predictions are recorded in Figure 13. Two SPEC predictions were performed differing only by the choice of the glassy shear modulus. These two values reflected the measured differences in moduli from the shear master curves shown in Figure 7 and were performed to demonstrate the parameter sensitivity of the model predictions. 


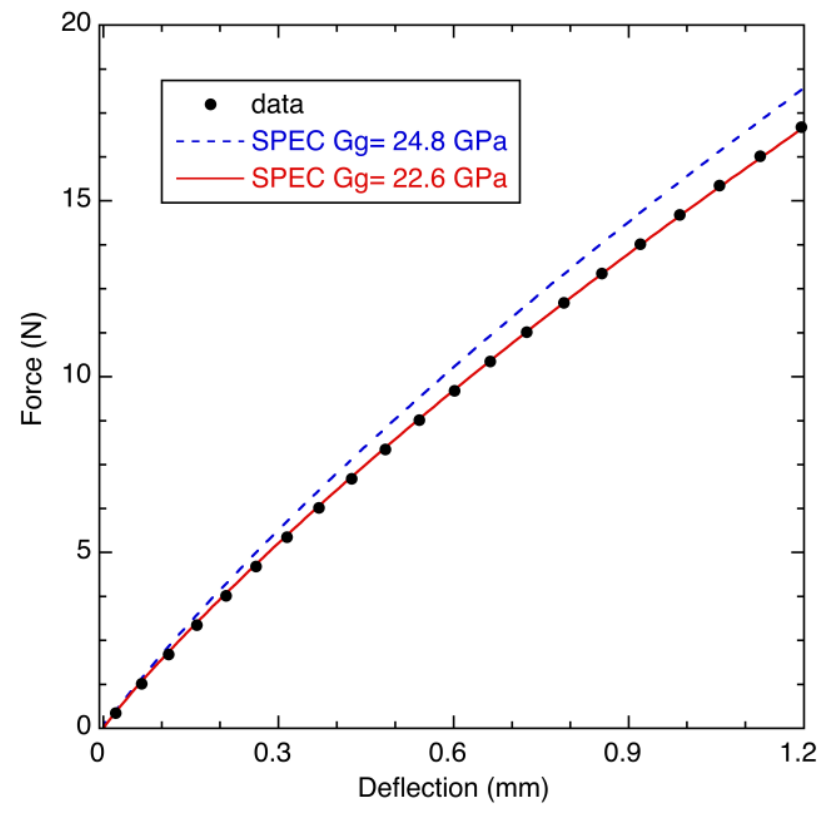

Figure 13 Comparison between measured deflection and SPEC predictions in 3-point bending test on $\mathrm{S} 8061$ glass beam loaded at $5 \mathrm{~N} / \mathrm{min}$ under isothermal conditions at $460^{\circ} \mathrm{C}$

The 3-point bending beam geometries also were tested in creep under several different magnitudes of loading. Annealed samples were reheated to $460^{\circ} \mathrm{C}$, held for 10 minutes and then the load was applied quickly (in seconds) while the mid-span creep deflection in the beam was measured as a function of time. The creep data and SPEC predictions are plotted in Figure 14.

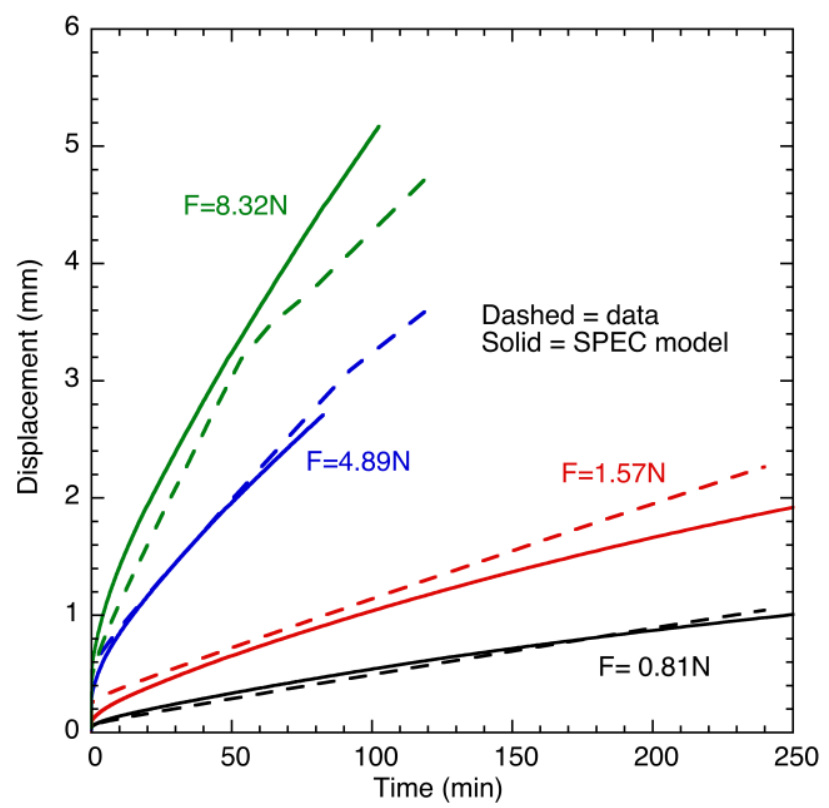

Figure 14 Measured deflections and SPEC model predictions from creep tests in 3-point bending of S8061 beams conducted at $460^{\circ} \mathrm{C}$ under four different loads 


\section{Discussion}

\subsection{Moduli temperature dependence}

The magnitude of the moduli measured in the dynamic torsion and 3-point bending tests are sensitive to the test specimen geometry. Variations in the thickness of sample or faces that are not parallel can affect the geometric factors that are used to calculate moduli. This does not, however, diminish the accuracy of the temperature dependence measured under small deformations. At higher temperatures, two issues can arise. One comes from slipping in the grips generated by the inherent mismatch in thermal strains between the glass and the grip materials. This can be alleviated by retightening grips at elevated temperatures. The second arises from the natural flow in the glass that occurs above glass transition. This was a problem encountered in the 3-point bending experiments as the shape of the beam began to change quite rapidly above $500^{\circ} \mathrm{C}$ limiting the data acquisition. However, the torsion test was able to record data successfully up to nearly $600^{\circ} \mathrm{C}$.

The observed variation in the storage moduli is about 5\%. Although it is difficult to conclude much about the effect of conditioning from such a small data sample, the cooling rate seems to produce a greater change in storage modulus than annealing. More test data are needed to confirm this as a finding. The loss moduli are equally consistent below $500^{\circ} \mathrm{C}$. There is the appearance of a relative maximum in the loss moduli between $200^{\circ} \mathrm{C}$ and $300^{\circ} \mathrm{C}$. This was unexpected since it occurs about $200^{\circ} \mathrm{C}$ below the glass transition temperature in a regime that was thought to be elastic. Nevertheless, this peak is reproducible being observed in every test conducted.

The other important information collected from these test results is the definition of the glass transition temperature. Although somewhat subjective in interpretation, the onset of glass transition occurred between $460^{\circ} \mathrm{C}$ and $470^{\circ} \mathrm{C}$.

\subsection{Shear master curves}

There are potential difficulties in building the master curves from data collected at the temperature extremes. At high temperatures, the glass may flow distorting the specimen shape and corrupting the geometric interpretation of moduli. At low temperatures, the structure is not equilibrated, and this can affect the material clock and shifting. Furthermore, at these temperature extremes, the frequency response curves provide limited data, making it more difficult to do the shifting required to generate the 
master curve. This contributes to the uncertainty in the glassy and equilibrium response. It is important to remember that these data reductions are an approximation from a thermorheological simplicity hypothesis being made to support engineering analyses. How well they work ultimately will determine the validity of the assumptions and model.

Noteworthy is the fact that the shear relaxation data taken directly from the $460^{\circ} \mathrm{C}$ torque relaxation experiment fall within the spread of the transformed oscillatory moduli in Figure 7. These curves represent findings from two independent tests measuring the same function. This greatly increases the confidence in the validity of the measured shear stress relaxation spectrum. The $10 \%$ differences between the dynamic torsion data (particularly noticeable at short times) come from several sources: variability in materials, variability in tests, assumed values for the glassy moduli and geometry differences. In mapping from the frequency to the time domain, there is always some ambiguity in the magnitude of the glassy response as the data are limited and the relaxation curves flatten out when approaching the tails at the beginning or end of the relaxation spectra. At those extremes, the limiting magnitudes become more uncertain. There are also subtle differences from sample to sample that influence geometric factors contributing to the interpretation of the modulus. However, when the normalized relaxation functions, $\mathrm{f}_{2}(\mathrm{t})$, are considered together, the differences among curves is reduced further, certainly to a range tolerable for engineering analyses.

\subsection{Thermal strain data}

Because the Netzsch dilatometer uses a small axial load to maintain contact between the probe and the sample during temperature cycling, mechanical deformations can distort the measurement of thermal strain. Although the load had no effect below the glass transition, at temperatures above $500^{\circ} \mathrm{C}$ the data did exhibit some noticeable creep under load. This was readily discernible by the reduction in the rate of expansion of the liquid on heating.

The SPEC fits to the thermal cycling data in Figure 8 and Figure 9 are quite good. The thermal expansion slopes in the glassy and liquid states are reproduced accurately as is the glass transition. During the slow reheat after a rapid cooling, the added structural relaxation is evident by the enhanced hysteresis near the glass transition temperature. The SPEC prediction shows the gap between cooling and heating curves narrowing at the lower temperatures, but it persists down into the $250^{\circ} \mathrm{C}$ range. This differs somewhat from the data where the hysteresis collapses and the curves come together in the $330^{\circ} \mathrm{C}$ 
range.

Perhaps the most difficult test for a nonlinear viscoelastic material model is one that involves multiple, reversing step histories. The solutions are path dependent, ratedependent and are influenced by the fading memory of all past history. That extreme test is the basis for choosing the time-temperature histories in Figure 11 and Figure 12. A noticeable trend is observable. Although the SPEC predictions are quite good during the cooling steps and temperature holds, the SPEC model has greater difficulty predicting the reversed heating steps. This effect is exacerbated by multiple, consecutive heating steps where the errors accumulate. Even though the relaxation rates during the temperature holds on reheating are quite reasonable, errors in the total strain change attributed to the step increase in temperature tend to push the differences between model and experiment further apart with each added step. Although this is a difference between model and data, its significance in residual stress analyses is less clear. Additional testing is needed to answer that question. Fortunately, the processing involved in predicting the residual stresses for many applications involves annealing and cooling without such temperature reversals. The multiple cooling steps with holds are predicted quite well.

\subsection{Monotonic loading and creep}

The SPEC model predictions for the 3-point bending tests were obtained from three-dimensional finite element analyses using the ADAGIO Sierra finite element code. The appropriate boundary conditions and loading rates were defined for each specific test. A regular mesh with six uniform gradient hexahedral elements through the thickness was employed for all computations. Contact conditions were modeled at the supports and loading point with an interfacial friction coefficient assumed to be 0.30 .

The SPEC analyses in Figure 13 employed identical relaxation spectra but two different values of the glassy shear modulus based on the variation seen in the dynamic master curves plotted in Figure 7. This provides useful information regarding the sensitivity of the model predictions to differences in the glassy shear modulus. Given the proper choice of the modulus, the prediction and data are virtually identical. The shape of the load-deflection curve is excellent, indicating the relaxation during loading is being captured accurately.

The final set of four tests predicted creep under different load levels. In each case, the comparison was quite favorable. A reproducible kink in the data curves was observed in the higher loaded samples at 3-3.5 mm of displacement. This was thought to be an experimental artifact of slip/stick in the contact conditions. 


\section{Conclusions}

The stress and volume relaxation associated with the solidification of an inorganic glass were analyzed using the nonlinear viscoelastic simplified potential energy clock (SPEC) model developed for glassy thermosets. The viscoelastic properties of Schott 8061 glass were determined by a combination of direct measurements and inferred behavior. Thermorheological simplicity was confirmed by construction of a master curve for the shear storage and loss moduli measured in oscillatory tests varying frequency at different isothermal temperatures. The bulk response was deduced by an iterative process choosing the relaxation function, moduli and thermal expansion coefficients to minimize the error between the SPEC finite element model predictions and the thermal strains measured under different temperature histories. Using this model calibration, additional tests were analyzed to assess the fidelity of the SPEC predictions. This included rigorous multi-step temperature histories with reversing ramp rates and prolonged holds to characterize the structural relaxation. Three point bending tests were conducted under monotonic loading ramps as well as creep under several different dead loads. In all cases, the analysis predictions fell within the bounds of what would be considered engineering accuracy for residual stress analyses in most applications.

The SPEC constitutive formalism is able to predict volume changes and relaxation without using a fictive temperature, thermal strain equation or structural relaxation function. This capability comes naturally from the role of the relaxation spectrum common to the bulk and temperature hereditary integrals defining stress. Indeed, when the bulk modulus is assumed to be constant, the spectrum of the temperature integral is seen to be identically equal to the Narayanaswamy structural relaxation function. Based on the findings to this point, it appears that the SPEC constitutive formalism is capable of predicting the solidification stresses and strains encountered in both organic and inorganic glass-forming materials. Moreover, the material characterization tests and model calibration procedures make this feasible for practical engineering applications.

\section{Acknowledgements}

Sandia National Laboratories is a multi-program laboratory managed and operated by Sandia Corporation, a wholly owned subsidiary of Lockheed Martin Corporation, for the U.S. Department of Energy's National Nuclear Security Administration under contract DE-AC04-94AL85000. 


\section{References}

1. O. S. Narayanaswamy, Ceram Trans 29 (1992) 315-328.

2. D. B. Adolf, R. S. Chambers, M. A. Neidigk, Polymer 50 (2009) 4257-4269.

3. O. S. Narayanaswamy, J. Am Ceram Soc 54 (1971) 491-498.

4. H. Leaderman, Elastic and Creep Properties of Filamentous Materials (1943) 175.

5. F. Schwarzl, A. J. Staverman, J Appl Mech 23 (1952) 838-843.

6. $\quad$ I. W. Morland, E. H. Lee, Trans Soc Rheol 4 (1960) 233-263.

7. A. Q. Tool, J Amer Ceram Soc 29 (1946) 240-253.

8. O. S. Narayanaswamy, J Amer Ceram Soc 64 (1981) 109-114.

9. J. H. Nielsen, J. F. Olesen, P. N. Poulsen, H. Stang, Comput Struct 88 (2010) 963972.

10. J. Kong, J. H. Kim, K Chung, Met Mater - Int 13 (2007) 67-75.

11. N. Siedow, T. Grosan, J Am Ceram Soc 88 (2005) 2181-2187.

12. J. H. Kim, J. Kong, K. Chung, Int J Mech Sci 66 (2013) 249-259.

13. N. Barth, D. George, S. Ahzi, Y. Remond, N. Joulaee, Mech Time-Depend Mater 18 (2014) 81-96.

14. W. G. Knauss, I. J. Emri, Comp Struct 13 (1981) 123-128.

15. W. G. Knauss, I. J. Emri, Polym Eng Sci 27 (1987) 86-100.

16. R. M. Shay, J. M. Caruthers, J Rheol 30 (1986) 781-827.

17. R. M. Shay, J. M. Caruthers, Polym Eng Sci 30 (1990) 1266-1280.

18. R. A. Shapery, Polym Eng Sci 9 (1969) 295-310.

19. P. A. O'Connell, G. B. McKenna, Mech Time-Depend Mater 6 (2002) 207-229.

20. J. M. Caruthers, D. B. Adolf, R. S. Chambers, P Shrikhande, Polymer 45 (2004) 4577-4597.

21. D. B. Adolf, R. S. Chambers, J. M. Caruthers, Polymer 45 (2004) 4599-4621.

22. M. L. Williams, R. F. Landel, J. D. Ferry, J Amer Chem Soc 77 (1955) 37013701.

23. Tina Hecksher, Niels Boye Olsen, Kristine Niss, Jeppe C. Dyre, J Chem Phys 133 (2010) 174514.

24. A. Jain, A. Y. Yi, J Am Ceram Soc 88 (2005) 530-535.

25. J. F. Ganghoffer, Mech Time-Depend Mater 4 (2000) 359-379.

26. T. F. Soules, R. F. Busbey, S. M. Rekhson, A. Markovsky, M. A. Burke, J Am Ceram Soc 70 (1987) 90-95.

27. G. W. Scherer, S. M. Rekhson, J Am Ceram Soc 65 (1982) 352-360.

28. S. M. Rekhson, O. V. Mazurin, J Am Ceram Soc 57 (1974) 327-328.

29. Sierra Solid Mechanics Team, Sierra/SolidMechanics 4.22 User's Guide, SAND2011-7597, October 2011

30. R. Gy, J Non-Cryst Solids 128 (1991) 101-108.

31. M. S. Rekhson, S. M. Rekhson, J Am Ceram Soc 69 (1986) 704-708.

32. R. D. Corsaro, Phys Chem Glasses 17 (1976) 13-22. 